\title{
A CONSTITUTIVE MODEL FOR STRUCTURED COAL BASED ON FRACTURE AND DAMAGE MECHANICS
}

\author{
KOU SHAOQUAN, $\uparrow$ YE DONGYING and DING YANSHENG \\ Institute of Mechanics, Academia Sinica, Beijing, People's Republic of China
}

\begin{abstract}
Considering the microcracks as the main factor influencing the mechanical properties of the structural coal this paper derives a system of equations to describe the mechanical behavior of structured coal under loading. In this connection, the structured coal exhibits three distinctive strengths, i.e. yield strength, limit strength and residual strength, and they split the whole deformation and failure process of the coal into four stages. A mechanical model for describing the strengths and the various failure stages is suggested. Triaxial tests are carried out in order to verify the model. Specimens are prepared with coal powder taken from one of the gas and coal outburst field in China. The test results are in reasonably good agreement with the theoretical ones.
\end{abstract}

\section{INTRODUCTION}

IN COAL and gas outburst a large amount of coal is fractured and thrown out together with gas within several tens of seconds. It is a very serious disaster in coal mining and has been studied for more than one hundred years. Recently, mechanists join the research team and some new knowledge about the burst mechanism has been obtained[1,2]. In this connection a constitutive model of burst coal is badly needed.

Most of the research works on coal behaviors were concentrated on its limit strength for a long time, for example[3]. Recently, Li paid attention to the influence of gas filling into pores on the stress-strain curves of coal, experimentally[4]. Unfortunately, the authors have not seen a discussion on the constitutive model for describing the whole deformation and failure process of coal, even without gas, yet. The present work is going to establish a model for describing the whole deformation and failure process of coal without gas from the point of view of the fracture and damage mechanics and the results could be a first step of studying the coal behavior with gas.

\section{BASIC EXPERIMENTAL PHENOMENA AND MAJOR ASSUMPTIONS}

A typical stress-strain curve of coal in triaxial compression is shown in Fig. 1. The stress and strain are defined as positive in compression state in the context. From the triaxial test results it is clear that:

(1) The relation between stress and strain is approximately linear below the stress $\sigma_{\mathrm{d}}$.

(2) Non-linear volumetric expansion respective to linear compression occurs under a stress higher than $\sigma_{\mathrm{d}}$ and finally the volumetric strain decreases with increasing stress.

(3) In the post failure region the specimen could bear a load but the bearing ability decreases with increasing strain.

(4) The bearing ability in the post failure region does not decrease to zero and it decreases to a certain value connected with the confining pressure instead. Afterwards the stress keeps nearly constant with increasing strain.

We shall start from the experimental facts. Corresponding to the features cited above there are three distinctive strengths which split the whole deformation and failure process into four stages and the strengths will be named as yield strength, limit strength and residual strength, respectively,

†Kou Shaoquan, Associate Professor, at the Beijing University of Iron and Steel Technology, and concurrently at the Institute of Mechanics, Academia Sinica. 


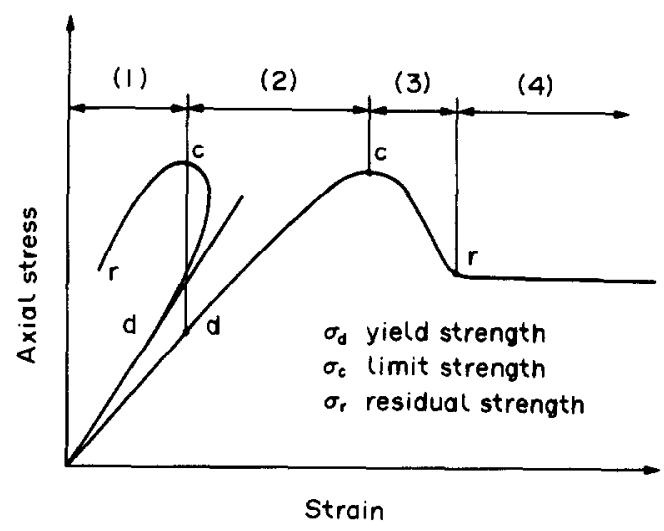

Fig. 1. Typical stress-strain curve of coal in triaxial compression for explaining the mechanical model. $\sigma_{\mathrm{d}}=$ yield strength, $\sigma_{\mathrm{c}}=$ limit strength $\sigma_{\mathrm{r}}=$ residual strength. (1) Elastic region (2) initial damage region,

(3) post failure region, (4) shearing and slipping region

and the four stages will be called elastic region, initial damage region, post failure region and shearing and slipping region, respectively.

Several basic assumptions will be made in the context.

(1) The specimen materials are initially isotropic and uniform in a comparatively large element. The coal material could be divided into two parts, matrix and cracks. The so called matrix includes solid coal and cementing materials. It is assumed that the matrix is linear elastic-perfect plastic body and Tresca criterion is used for describing its yielding.

(2) It is assumed that the mean stress tensor $P^{\prime} \delta_{i j}$ and the deviatoric stress tensor $S_{i j}{ }_{i j}$, in the sense of normal continuum mechanics, are linearly related to the effective stress tensors $P \delta_{i j}$ and $S_{i j}$, respectively, which are defined in a comparatively larger element including cracks investigated when discussing the initial extension of preexisted cracks, i.e.

$$
\begin{gathered}
P^{\prime}=H_{1} P \\
S^{\prime}{ }_{i j}=H_{2} S_{i j}
\end{gathered}
$$

where $H_{1}$ and $H_{2}$ are parameters depending on the local characteristics of the material, including the influence of cracks and therefore, they are random variables.

(3) Crack volumetric density $\eta$ is defined, similar to ref. [5], as

$$
\eta=\lim _{\Delta V \rightarrow \Delta V_{0}} \frac{\Delta V_{\mathrm{v}}}{\Delta V}
$$

where $\Delta V_{v}$ is the void volume in $\Delta V$ and $\Delta V_{0}$ is a volume of the comparatively large element. It is assumed that the established relation between crack volumetric density and Poisson's ratio by Budiansky and O'Connell[6] is valid.

(4) The shear band in the specimen, once formed, splits the specimen into two parts and due to stress relieving, each of them may be treated as rigid and the specimen deformation is induced by the relative slip between the two rigid parts.

Other secondary assumptions will be stated in the following text.

\section{YIELD STRENGTH}

As pointed out previously, the coal behaves as linear elasticity below a threshold stress $\sigma_{\mathrm{d}}$. The elastic moduli, then, could be obtained by considering the matrix and the preexisted cracks, given by Walsh[7], or experimentally.

A lot of experiments in rock mechanics, for example[8], show that among all of the stress-strain curves the curve $\sigma_{1}-\epsilon_{\theta}$ deviates from linearity earliest, where $\sigma_{1}$ and $\epsilon_{\theta}$ are, respectively, the axial stress and circumferential strain. This is corresponding to the fact that the observed microcracks by microscopy in the sliced specimen are parallel to the major compression stress at 
the beginning of dilatancy. Crack growth and nucleation in specimen induce dilation. The onset of dilatency could be corresponding to axial extension of pre-existed cracks in mode I when we limit ourselves to discuss a case of axial stress greater than or equal to confining pressure.

If there is a penny crack with a unit vector $\mathbf{n}$ in its normal direction, and a characteristic diameter $2 c$ and a uniform tensile stress $\sigma_{\mathrm{n}}$ acted on the crack plane, the stress intensity factor can be written as

$$
K_{\mathrm{I}}=\frac{2}{\sqrt{\pi}} \sqrt{c} \sigma_{\mathrm{n}}
$$

and $\sigma_{\mathrm{n}}$ could be expressed in terms of the stress tensor in the proximity of the crack as

$$
\sigma_{\mathrm{n}}=P^{\prime} \delta_{i j} n_{j}+S_{i j}^{\prime} n_{j}
$$

where $n_{j}$ is the $j$ th component of $\mathbf{n}$. However, according to the assumption (2)

$$
P^{\prime} \delta_{i j} n_{j}+S_{i j}^{\prime} n_{j}=H_{1} P \delta_{i j} n_{j}+H_{2} S_{i j} n_{j}
$$

In an axially symmetrical problem, when

$$
\frac{2}{\sqrt{\pi}} \sqrt{c}\left(H_{1} P+H_{2} \tau\right)=K_{\mathrm{lc}}
$$

axial extension of a crack with a diameter of $2 c$ and normal direction perpendicular to the axis would occur, where $\tau$ is the shear stress, $K_{\mathrm{Ic}}$ is the critical stress intensity factor depending on the local characteristics of the material. Obviously, $K_{\mathrm{lc}}$ is a random variable.

As we know, the crack length should be also a random variable. Corresponding to the onset of dilatency neither one crack extends nor all of the cracks extend axially. It is proposed that, corresponding to the onset of dilatancy, the expectation values of these random variables fulfil eq. (5), i.e.

$$
\left.\frac{2}{\sqrt{\pi}} \overline{\left(H_{1} \sqrt{c} P\right.}+\overline{H_{2} \sqrt{c} \tau}\right)=\overline{K_{\mathrm{lc}}}
$$

where the parameters with bars over them represent their individual expectation values and they are the material constants, including the influence of crack size and distribution. Since

$$
\tau=\frac{1}{2}\left(\sigma_{1}-\sigma_{3}\right), \quad P=\frac{1}{3}\left(\sigma_{1}+2 \sigma_{3}\right) .
$$

Equation (6) becomes

or

$$
\frac{1}{2}\left(\sigma_{1}-\sigma_{3}\right)=-\frac{\overline{H_{1} \sqrt{c}}}{3 \overline{H_{2} \sqrt{c}}}\left(\sigma_{1}+2 \sigma_{3}\right)+\frac{\sqrt{\pi}}{2} \frac{\overline{K_{\mathrm{lc}}}}{\overline{H_{2} \sqrt{c}}}
$$

$$
\left(1+\frac{2 \overline{H_{1} \sqrt{c}}}{3 \overline{H_{2} \sqrt{c}}}\right) \sigma_{1}=\left(1-\frac{4 \overline{H_{1} \sqrt{c}}}{3 \overline{H_{2} \sqrt{c}}}\right) \sigma_{3}+\frac{\sqrt{\pi} K_{\mathrm{lc}}}{\overline{H_{2} \sqrt{c}}}
$$

where $\sigma_{1}$ and $\sigma_{3}$ are axial stress and confining pressure, respectively. Equation (7a) or (7b) describes the initial damage curve, or the so called initial yield, in the stress space. Over this curve crack growth and nucleation occur but below it there is no damage and the elastic relation is tenable.

\section{INITIAL DAMAGE REGION AND STRENGTH}

Let $N$ represent the crack number in $\Delta V, a$ is a certain crack size and $\overline{a^{3}}$ is the ratio of average crack volume to $\Delta V$, then

$$
\eta=\gamma N \overline{a^{3}}
$$

where $\gamma$ is a constant for adjusting the nonspheric characteristic of the crack. The crack growth is described by

$$
\overline{a^{3}}=\beta \epsilon_{\mathrm{v}}^{l}
$$


where $\beta$ is a non-dimensional constant depending on the fracture toughness, bulk modulus, grain size and Poisson's ratio of the matrix, $\epsilon_{\mathrm{v}}$ is the volumetric tensile strain $\epsilon_{\mathrm{v}}=\epsilon_{i j} \delta_{i j}$, and $l$ is a constant to be determined. The crack nucleation is described[9] by

$$
N=\alpha \epsilon_{\mathrm{v}}^{m} .
$$

Generally, crack nucleation and crack growth are influenced by loading rate and then

$$
\dot{\eta}=K_{\mathrm{I}} \epsilon_{\mathrm{v}}^{n-1} \dot{\epsilon}_{\mathrm{v}}
$$

where

$$
K_{1}=\gamma \beta \alpha(m+l), \quad n=m+l .
$$

According to the assumption (3) the relationship obtained by Budiansky and O'Connell[6] could be valid in this case except a constant

$$
\eta=\frac{K_{2}\left(v_{m}-v\right)}{(1+v)\left[10 v_{m}-v\left(1+8 v_{m}\right)\right]} .
$$

Figure 2 shows the one dimensional compression test results for Stripa granite, where $G$ and $G_{50}$ are the shear modulus at $\epsilon_{\theta}$ and that at half of the limit strength, respectively. Referencing Fig. 2 it might be reasonable to assume that

$$
G / G_{m}=1-D
$$

where and thereafter a letter with a subscript $m$ describes the corresponding property of the matrix. $D$ is a damage variable and considering the relation between shear modulus and Poisson's ratio[6] the damage variable $D$ could be written as

$$
D=f(v) \eta
$$

where

$$
f(v)=K_{3}+K_{4} v
$$

$K_{3}$ and $K_{4}$ are constants to be determined. Then

$$
\dot{D}=\left[f(v)+\eta \frac{\partial f}{\partial v} \frac{\partial v}{\partial \eta}\right] \dot{\eta}
$$

and

$$
\frac{\partial v}{\partial \eta}=\frac{-K_{\mathrm{I}}(1+v)^{2}\left[10 v_{m}-v\left(1+8 v_{m}\right)\right]^{2}}{(1+v)\left[10 v_{m}-v\left(1+8 v_{m}\right)\right]+\left(v_{m}-v\right)\left[10 v_{m}-\left(1+8 v_{m}\right)(1+2 v)\right]}
$$

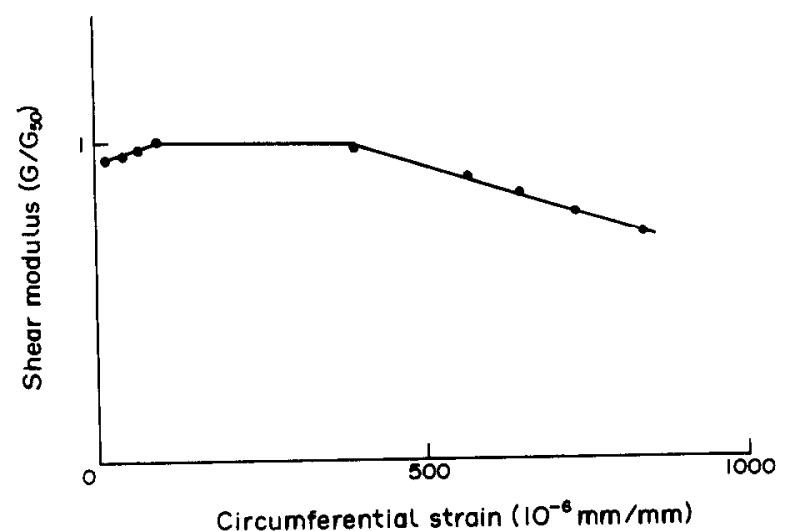

Fig. 2. Relation between shear modulus and circumferential strain for Stripa granite in uniaxial compression. 
With linear damage assumption, the following relations could be obtained

$$
\begin{aligned}
P & =3 K_{m}(1-D) \epsilon_{i j} \delta_{i j} \\
S_{i j} & =2 G_{m}(1-D) e_{i j}
\end{aligned}
$$

where $e_{i j}$ is the deviatoric strain. Equations (11), (12), (15), (16) and (18) describe the relationship between stress and strain in initial damage region.

The above analysis is based on and developed from ref. [9]. According to the assumption that the matrix is linear elastic before yielding eq. (18) is valid when the stress is both greater than the onset of dilatancy and less than the limit strength. The stress of the cracked body reaches the limit strength when the matrix is yielded or the stress of the cracked body decreases with increasing strain due to growth of cracks if the matrix is still elastic. The latter case will not be discussed in the present paper. The limit strength should be in accordance with the Mohr envelope of cracked body obtained by experiment. Therefore the constants could be determined.

\section{RELATION IN POST FAILURE REGION}

After the yield of matrix, on the one hand, the bearing capacity of the matrix in unit area keeps constant, whatever how large the strain is. On the other hand, due to crack rapid growth and crack nucleation with increasing deformation the bearing area of the matrix is reduced with gradually forming of shear band. Hence, the following relation is fulfilled

$$
\left(A-A_{\mathrm{s}}\right) g(\mu, \theta) \sigma_{3}+A_{\mathrm{s}} \sigma_{\mathrm{s}}^{*}=A \sigma_{1}
$$

where $A_{s}$ and $A$ are bearing area of the matrix and the cracked body, respectively, $\mu$ is the friction coefficient and $\theta$ is an effective angle between crack plane and $\sigma_{3}$. The function $g(\mu, \theta)$ is introduced for describing the friction between crack surfaces. $\sigma_{\mathrm{s}}^{*}$ is the yield strength of the matrix. With the linear damage assumption

$$
A_{\mathrm{s}}=A_{\mathrm{s}}(1-D)
$$

and

$$
A=\frac{A_{\mathrm{s}}^{0}}{1-\eta_{0}}\left(1+v \epsilon_{1}\right)^{2} .
$$

Hence, when $D$ is comparatively small the first term in eq. (19) could be neglected and therefore

$$
\sigma_{1}=\frac{(1-D)\left(1-\eta_{0}\right)}{\left(1+v \epsilon_{1}\right)^{2}} \sigma_{s}^{*}
$$

where $\eta_{0}$ is the initial crack density. Strain rate and confining pressure will have influence on the stress-strain curve in the present region through their influence on $D$ and the onset of dilatancy.

\section{RESIDUAL STRENGTH AND THE SHEARING AND SLIPPING REGION}

Once the macro shear bands is formed the cemented coal structure within the band is, partly or totally, disintegrated by the densely populated cracks and the materials outside the band are stress relieved due to the formation of the band. $D$ is no longer small. The second term in eq. (19) could be neglected since $A_{\mathrm{s}}$ approaches to zero and the problem could be, then, simplified by assuming two rigid bodies to slip with each other with small coal grains in between. Therefore

$$
\tau=\mu \sigma_{\mathrm{n}}
$$

where $\tau$ is the friction force in unit area parallel to the shear band and $\sigma_{n}$ is the normal stress. For axially symmetric problem

$$
\sigma_{1}=\frac{\operatorname{tg} \theta(\cos \theta+\mu \sin \theta)}{(\sin \theta-\mu \cos \theta)} \sigma_{3}
$$

where $\theta$ is the angle between shear band and $\sigma_{3}$. 


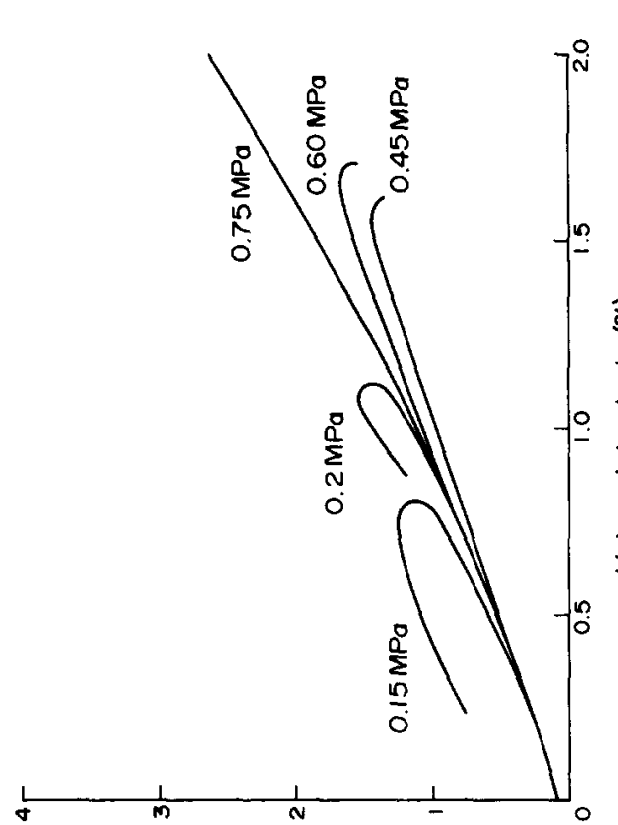

(DdW) ssarzs po!quanaph!o
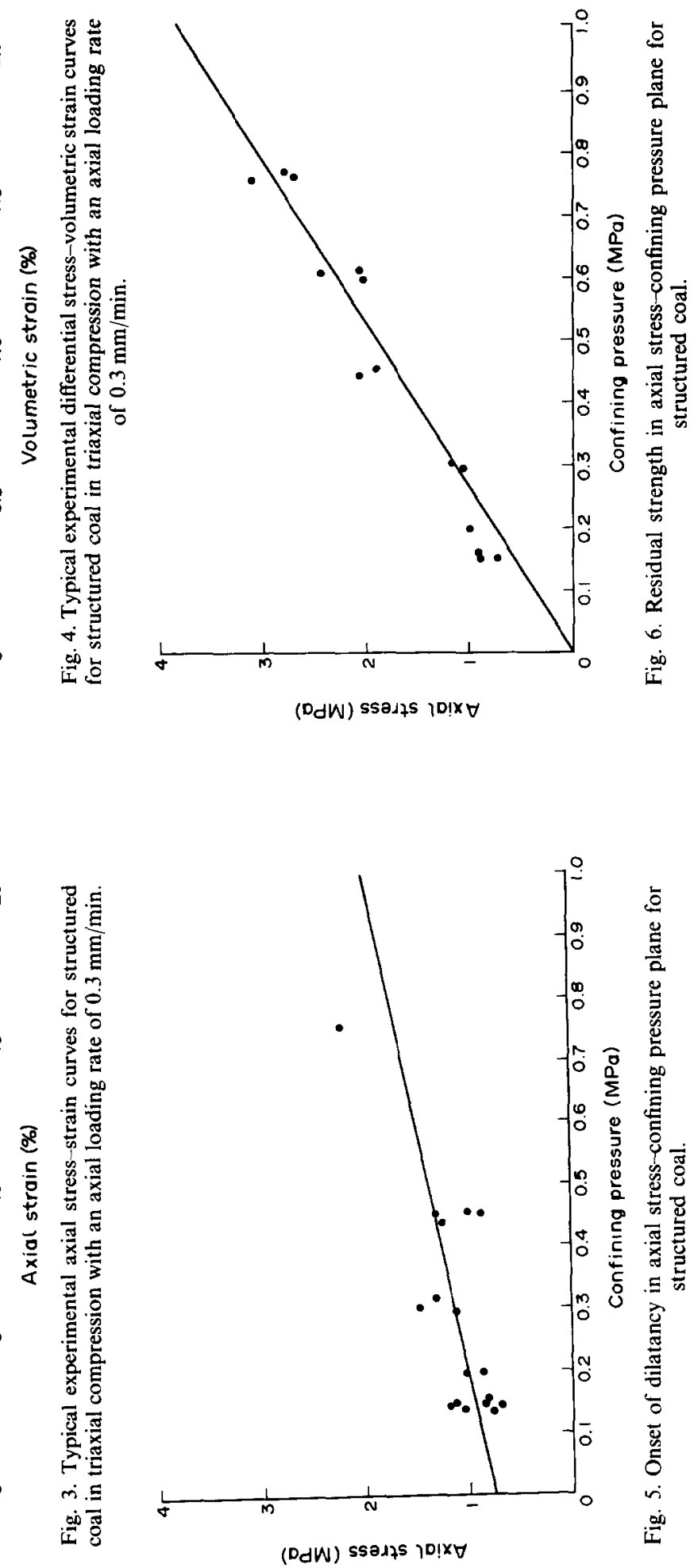
Equation (24) becomes (25) when $\theta=45^{\circ}$

$$
\sigma_{1}=\frac{1+\mu}{1-\mu} \sigma_{3}
$$

Equation (24) or (25) is the relation between residual strength and confining pressure. Thereafter the axial stress keeps constant with the increasing strain approximately, as if a perfect plasticity material behaves.

\section{EXPERIMENTAL VERIFICATION}

In order to verify the theoretical results, triaxial tests with structured coal specimens are carried out. Specimens are prepared with coal powder taken from one of the coal and gas outburst fields in China, the Beipiao Coal Mine. The coal powder with grain size of $0.1-0.2 \mathrm{~mm}$ is firstly homogeneously mixed with $17 \%$ of water, then compacted into specimen shape, finally put in a temperature environment of $105^{\circ} \mathrm{C}$ for $9 \mathrm{~h}$ and that makes the so-called structured coal specimen. The tests are carried out at room temperature. Although field coal varies from place to place in formation conditions, such as tectonic movement, weathering and present tectonic pressure, etc., the structured coal might represent soft coal. Specimens are tested in a $10,000 \mathrm{~kg}$ Stepless Compression Test Machine made by the WF Company (England), with constant confining pressure and loading rate during the test. Axial load and displacement, confinding pressure and volumetric change are measured. Programs are made for recording and processing the data during tests in terms of a computer, Hewellet Packard. The whole experimental results will be published elsewhere[10]. The strain rate and confining pressure have influence on the mechanical properties of the specimens. Figures 3 and 4 are the axial stress-strain, differential stress-volumetric strain relations under various confining pressure with axial loading rate $0.3 \mathrm{~mm} / \mathrm{min}$, corresponding to a strain rate $(2-6) \times 10^{-5} / \mathrm{s}$. The general trend of experimental curves is in a reasonable good agreement with the theory by judging these two figures. However, the brittleness of the specimen is reduced when the confining pressure increases. The dilatancy would not occur any more when the confining pressure reaches $0.45 \mathrm{MPa}$ from the figures and then, the failure of the specimen becomes ductile. Therefore the previous analysis is suitable only on the case under lower confining pressure.

Equation (7a) or ( $7 b$ ) shows that the relation between shear stress and mean stress at the onset of dilatancy should be a straight line with the same expectation of the crack size and distribution parameters and the same expectation of fracture toughness for different specimens. The slop of the line is dependent on the characteristics of the crack size and distribution. The intercept is not only depending on the crack characteristics but also on the expectation of the fracture toughness. Therefore from eq. (7a) the line would be concaved if $K_{\mathrm{lc}}$ increases significantly with increasing confining pressure[11]. The experimental results with the above mentioned artificial specimens manifest themselves a rather good straight line between $\sigma_{1}$ and $\sigma_{3}$ at the onset of dilatancy (Fig. 5) with a regression coefficient of 0.83 .

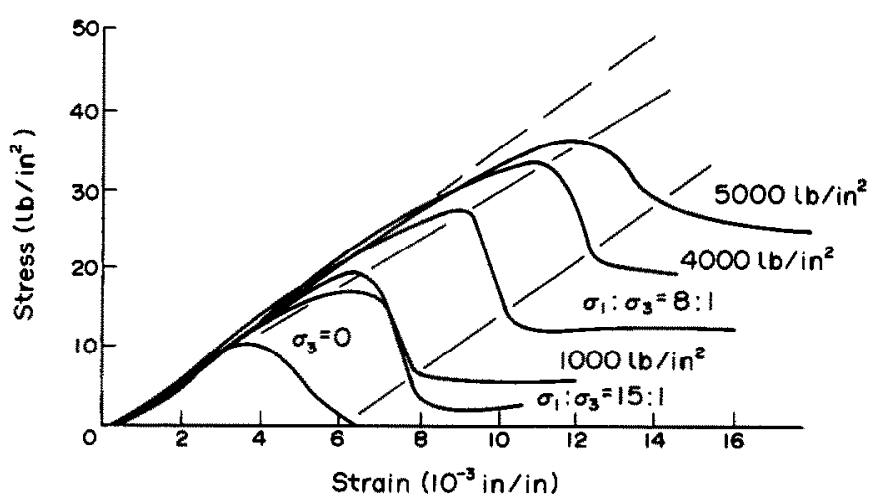

Fig. 7. Typical axial stress-strain curves for sandstone in triaxial compression[12]. 


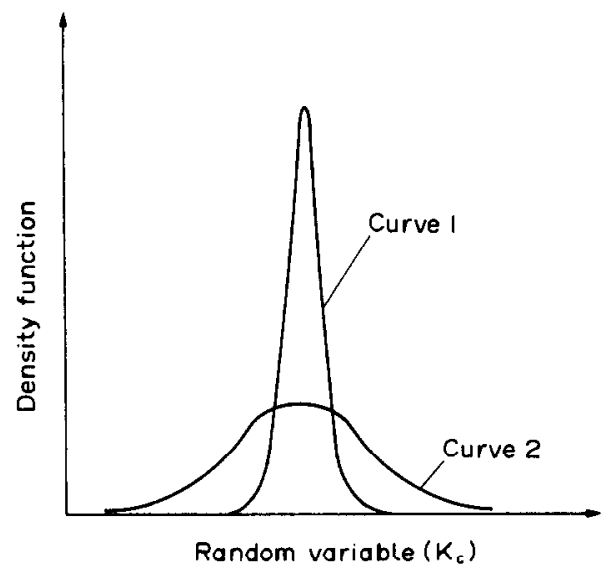

Fig. 8. Two kinds of typical distribution density function of the random variable $K_{\mathrm{c}}$.

Equation (24) describes a linear relation between residual strength and confining pressure when the fracture angle and friction coefficient keeps constant. This is verified by the experimental results and in Fig. 6 the regression coefficient is 0.97 .

Sometimes the residual strength decreases slowly with increasing axial strain. This is explained as the small coal block within the shear band fractured gradually and continuously to smaller powder.

Quantitative discussion on the agreement between the theory and the experiment in the damage and the post failure regions will be given in the near future.

\section{DISCUSSIONS}

Microcrack growth and nucleation are the main failure mechanism of the structured coal. Initial yielding (onset of dilatancy), limit strength and residual strength divide the failure process of structured coal into four stages. The mathematical descriptions are different at different stages.

There is no significant difference between structured coal and brittle rock from the point of view of the microcrack growth and nucleation and the scheme of the above theory should be suitable to brittle rock, too. It is true by judging Fig. 7[12].

The failure mechanism of the post failure region of the structured coal is the intersection of macrocracks. The bearing area of the matrix decreases gradually with the growth of shear band. The extension of macrocrack might have two typical cases. One is that the local crack nucleation is liable to develop long cracks and the long cracks intersect with each other and make the bearing capacity of the specimen decrease if the fracture toughness in the specimen is comparatively uniform, i.e., the density function of the random variable $K_{\mathrm{c}}$ is more or less similar to Kronecker delta function just like curve 1 in Fig. 8. In the case the $\sigma-\epsilon$ curve decreases sharply. The specimen is brittle and the coal burst is liable to occur in the field with a fracture toughness distribution density function of curve 1 . The other is that the crack nucleation is not prone to develop a long crack since $K_{\mathrm{c}}$ along the expected crack path varies significantly if the values of fracture toughness in specimen spread widely, i.e., the density function of $K_{\mathrm{c}}$ is corresponding to the curve 2 in Fig. 8 . Therefore the structure failure is mainly due to the crack combination and $\sigma-\epsilon$ curve decreases slowly. Coal burst is not prone to occur in the field with a fracture toughness density function of curve 2 in Fig. 8.

Acknowledgements - This work is supported by The National Natural Science Foundation of China under contract No. 1850001. The authors wish to acknowledge the value of creative discussions with Prof. Zheng Zhemin, Prof. Tan Qinming, Prof. Yu Shanbing and the other members in the gas and coal outburst research group. Gratitudes are also expressed to the Mining Research Institute, Beijing University of Iron and Steel Technology, the Geotechnical Engineering Division of the Institute of Mechanics Academia Sinica and the Beipiao Coal Mine for their kindest supports to this work. 


\section{REFERENCES}

[1] Zheng Zhemin, A study on the mechanism of gas and coal outburst from the dimensional analysis viewpoint, in Mechanics and Production Construction. Beijing University Press, Beijing (1982).

[2] Yu Shanbing, Steady advance of coal gas bursts. Acta Mech. Sinica. 4, 77-85 (1988).

[3] W. H. Walton (Ed.), Mechanical Properties of Non-metallic Brittle Materials. pp. 5-28, 123-145. Butterworth, London (1958).

[4] Li Zhongcheng, Study on mechanism of coal and gas outburst in in-seam drivage. Chinese J. Coal 1, 17-27 (1987).

[5] Kou Shaoquan and Alm, Ove, Effect of microcracks on the tensile strength of granite. Proc. 2nd Congr. on Structural Failure, Product Liability and Technical Insurance, pp. 709-719. Interscience Publishers, London (1987).

[6] B. Budiansky and R. J. O'Connell, Elastic moduli of a cracked solid. Int. J. Solids Struct. $1781-97$ (1976).

[7] J. B. Walsh, The Effect of cracks on the uniaxial elastic compression of rocks. J. Geophys. Res. 70, 399-411 (1965).

[8] Kou Shaoquan, Yu Jiling and Young Genhong, An experimental study of the attenuation mechanisms of the stress wave propagating along a limestone bar. Acta Mech. Sinica 6, 582-589 (1982).

[9] J. S. Kuszmaul, A new constitutive model for fragmentation of rock under dynamic loading. Proc. 2nd Int. Symp. On Rock Fragmentation by Blasting, Keystone U.S.A., pp. 412-424 (23-26 August 1987).

[10] Ye Dongying et al., An experimental study on the mechanical properties of coal without gas. Research Report, Institute of Mechanics, Academia Sinica (1988).

[11] R. A. Schmidt and H. P. Rossmanith, Basic of rock fracture mechanics, in Rock Fracture Mechanics (Edited by H. P. Rossmanith). CISM Italy (1983).

[12] Z. T. Bieniawski, Propagation of brittle fracture in rock. Basic and Applied Rock Mechanics, Proc. 10th Symp on Rock Mechanics, Texas, U.S.A. Chapt. 15, p. 409. (1968). 